\title{
Sensitivity Analysis of Spray Painting Process to Input Parameters: Validation of CFD Jet Impingement Model against an Experimental Dataset
}

\author{
Barry Moore, Farhad Nabhani, Vahid Askari \\ School of Science \& Engineering \\ Teesside University \\ Middlesbrough, TS1 3BA, \\ United Kingdom
}

\begin{abstract}
Accurate modelling of the automotive spray paint process requires using Computational Fluid Dynamic (CFD) simulations built upon a number of interacting physical models. This study examines one component of the air flow in such a simulation - impinging single jet air flow and models the results in a generalizable form to allow comparison with existing practical experiment, validating the results and allowing the development of more complex models based upon reliable results. The results in this paper compare the data produced by four variations of mesh design and wall function using the $k-\varepsilon$ model with those produced from previous practical experimental data collected in the European Research Community on Flow (ERCOFTAC) database. The CFD data for each model shows good agreement with the experimental data set in and around the boundary layer close to the jet with particularly good results using the enhanced wall treatment and a fine resolution mesh with a $y+$ (dimensionless wall distance) value of 1 . Further refinements are required to fully resolve larger radial distances, however the general results produced show good agreement with expected phenomena.
\end{abstract}

\section{KEYWORDS}

CFD, Experimental, Validation, Paint, Spray, Robotics

\section{INTRODUCTION}

This research paper forms part of a larger study and aims to lay foundations for further research on accurately predicting spray paint deposition rate based upon a set of key parameters; the overall goal of the study being to understand the sensitivity of the process to each individual parameter through CFD modelling. This individual paper is intended to study the effectiveness of commercial CFD code at modelling a single jet of air impinging on a surface, creating wall bounded flow (one of the key variables affecting spray paint deposition.) By comparing the results from a number of models within the ANSYS Fluent commercial Computational Fluid Dynamics (CFD) package against previous practical results, the validity of the data can be tested and its reliability established for future work: namely establishing the sensitivity of the spray painting process as a whole to its various input parameters.

\subsection{BACKGROUND to AUtOMATED SPRAY PAINTING}

Paint finish quality is of high importance in the automotive industry and across other sectors. Being able to sell high value products requires a consistent, even \& blemish free surface with aesthetic appeal, however the quality of a painted coating (and in particular, the thickness of the coating) has other impacts in manufacturing processes, product life cycle and fixed and variable production costs. While it is easy for a casual observer to estimate the impact of an insufficiently thick paint coating (Poor visual appearance, inadequate corrosion resistance), the effects of a thicker than optimum coating are of equal or indeed greater consequence. Visual

Corresponding Author: Prof. F.Nabhani, Teesside University, Middlesbrough, TS1 3BA, United Kingdom Tel: +44 (0) 1642 342482, Mobile: +44 (0)7584 490272, email: f.nabhani@tees.ac.uk 
defects such as paint drips, runs and curtains occur when gravity driven flows pull paint downwards resulting in quality control rejects and rework (and potentially customer dissatisfaction,) while unseen defects can cause problems later in the products life cycle. Industrial drying processes are often calibrated to a particular wet film thickness based on the time taken for the volatiles in the paint to evaporate. When a paint layer is thicker than expected a layer of wet paint will often remain trapped under the surface layer resulting in defects that arise after a product reaches the customer. Insufficiently dry paint also prevents subsequent manufacturing processes from taking place for fear of damaging the wet paint - increasing lead time. Thicker paint coatings also increase variable costs - with an illustrative high solid paint coating having an optimum thickness of around 50 microns - an increase of the same small amount doubles material costs, delays further production and potentially incurs rework or customer returns/dissatisfaction.

Due to the factors listed above, minimizing the variation in spray coating thickness is a major requirement of the automotive industry with automated robot spraying used to provide consistent and precise results. Historically a process of trial and error was used to optimise the various input parameters (air pressures, distances, viscosities etc.) however CFD modelling has shown an increasing capability for modelling these processes with improving levels of accuracy.

\subsection{Simulation of Spray Paint Deposition (General)}

Due to the motivations discussed above, significant research into predicting the localised rate of paint deposition in industrial spraying has already been carried out, with several sub-areas of study appearing. Generally speaking, the input parameters responsible for determining paint deposition rate can be mapped as in in figure 1 [1-7]. Leaving aside the geometry of the set up (which can be easily varied), examining the process below it can be seen that the breakup (and subsequent deposition of paint particles) is dependent on air flow pattern and speed and the rheological properties of the paint and it is these areas that most of the

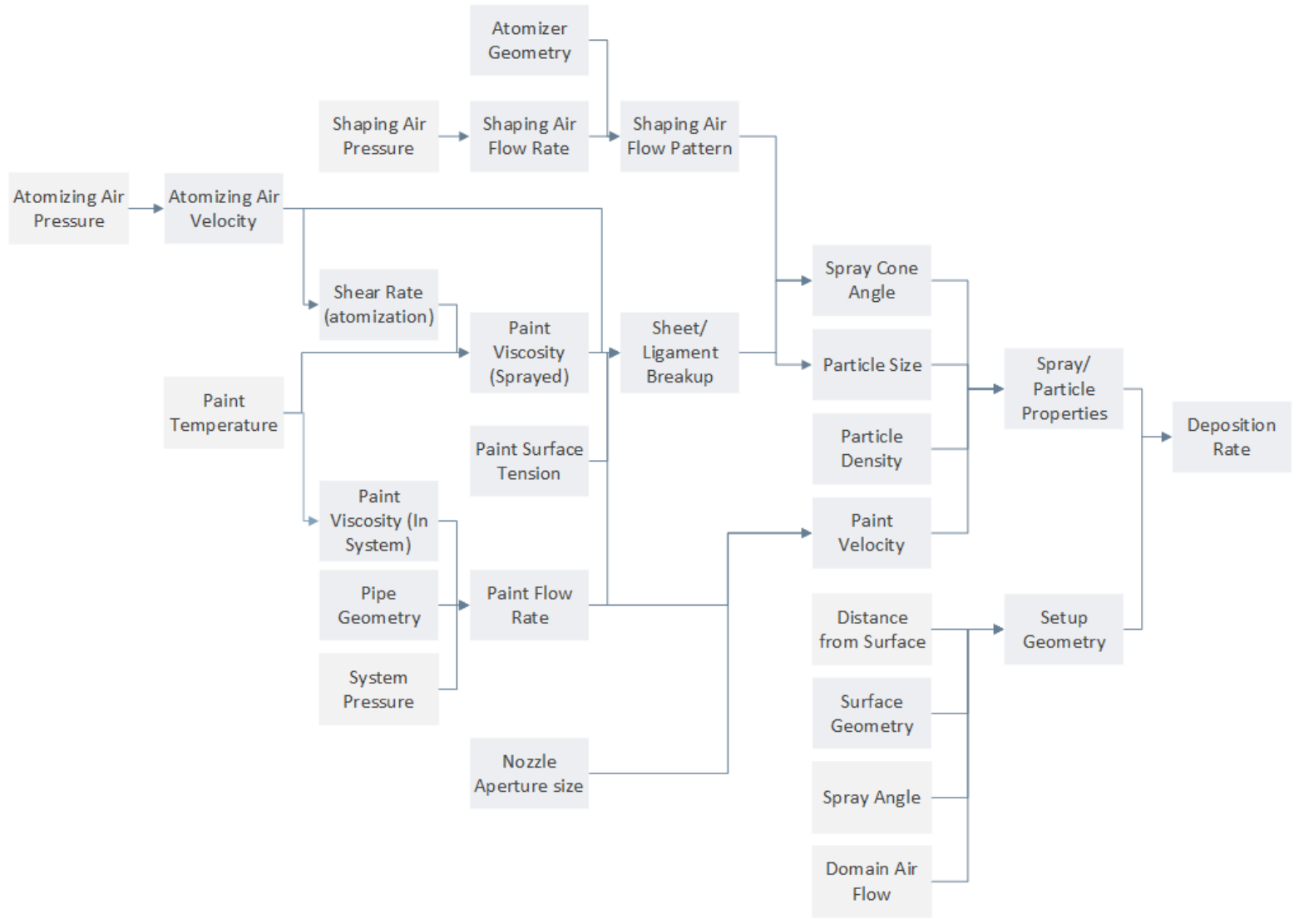

Figure 1 Map of spray painting variables 
existing literature is focused.

The work of $[1,3,5,8]$ investigate the effect of differing atomizer design (with differing atomisation or breakup mechanisms), while $[2-7,9,10]$ investigate the effect of ambient operating conditions along with paint rheology (although consensus does not appear to have been reached on the effect of paint thixotropy). The research of $[11,12]$ make comprehensive attempts to understand the sensitivity of the whole process to each input parameter; however it remains difficult to properly generalise the results due to the number of variables (and complexity of others such as atomiser geometry).

In almost of all of the current research and industrial application CFD is utilised to effectively model complex flow fields where it would be difficult to obtain experimental result however it is important to ensure the validity of the applied CFD model.

This study focuses on validation of one component of a whole CFD model used to model spray paint deposition. Complete models are comprised of complex continuous and discrete phase models (and the interaction between these two phases) along with assumptions about geometries and rheological properties; however validating these flows with experimental data is problematic. While the study at large hopes to understand the importance that each variable has in affecting the spray painting process, this paper attempts to validate one of the most significant component of the larger model, in this case a single high velocity air jet used to atomize the spray paint impinges on a surface normal to the jet.

\subsection{MOdeLLING OF Jet IMPINGEMENT}

When modelling the effect of air flow on deposition rate, the primary consideration is the trajectory of the particles transported within that flow $[8,13]$ - hence the velocity of the flow at a point needs to be measured. While many studies exist relating to CFD simulation of spray deposition, most studies of jet impingement relate to applications in heat transfer (heating \& cooling) and aerodynamics (vertical take-off applications) however many of the studies can be considered to be analogous and used for comparison of outputs. While interested primarily in heat transfer, the works of [14-17] use simulated or experimental setups with appropriate geometries and Reynolds numbers along with measurements of air velocity. In agreement with prescribed practice [18], [15] finds that the standard $\mathrm{K}-\varepsilon$ model fails to properly resolve the boundary layer turbulence under predicted near wall and over predicting at the outer layer; use of the $k-\varepsilon-v^{2}$ model or the newer realizable $\mathrm{k}-\varepsilon$ model is recommended.

\section{MATERIAL \& Methods}

The objective of this study is to use the recommendations of [15] relating to CFD modelling of Impinging jet flow when applied to the experimentally recorded data produced by $[17,19]$ with the recorded data contained within the open 'European Research Community on Flow' (ERCOFTAC) database[20]. Figure 2 shows the basic geometric setup of the simulation: 


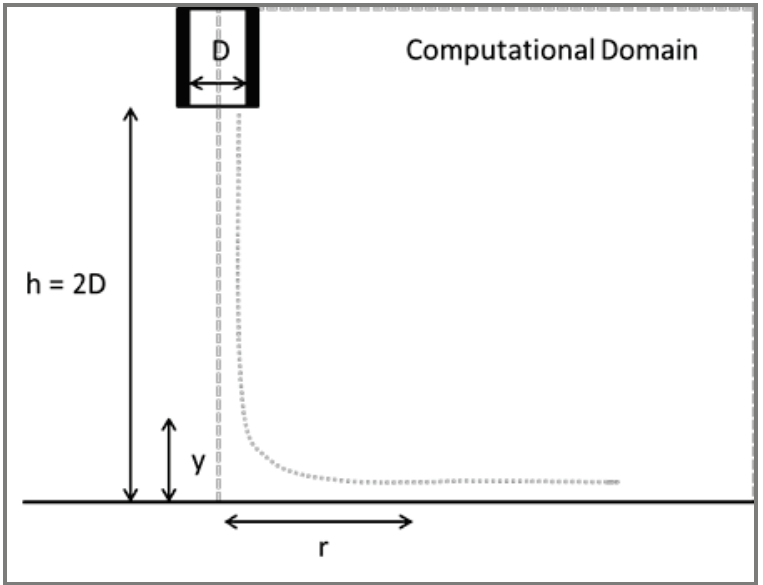

Figure 2: CFD model simplified setup

Inlet diameter $D$ is fixed at $101.6 \mathrm{~mm}$ with a distance from the inlet (nozzle) to the target plate of 2D $(203.2 \mathrm{~mm})$ to match the largest available volume of data within the experimental dataset [20]. While this value of $D$ and ratio of $h / D$ would be unusual in a spray painting setup, the results are presented in their generalized form (ratios of translation in a normal or parallel distance to the plate in terms of inlet diameter) and are valid for smaller values of $D$ and larger $h$ to $D$ ratios. A single stream of air at constant velocity is expelled from the inlet/nozzle (figure 3 ) and impinges on the target plate spreading out parallel to the plate (figure 4). The Reynolds number of the jet at inlet is 23,000 and pressure outlets of the model are at a distance of $>10 \mathrm{D}$ from the centre of the jet.

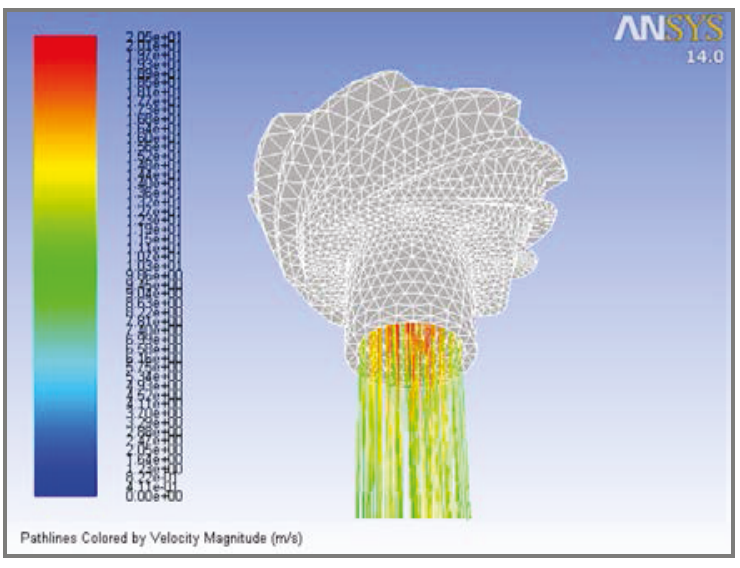

Figure 3: Swirl nozzle with central air stream

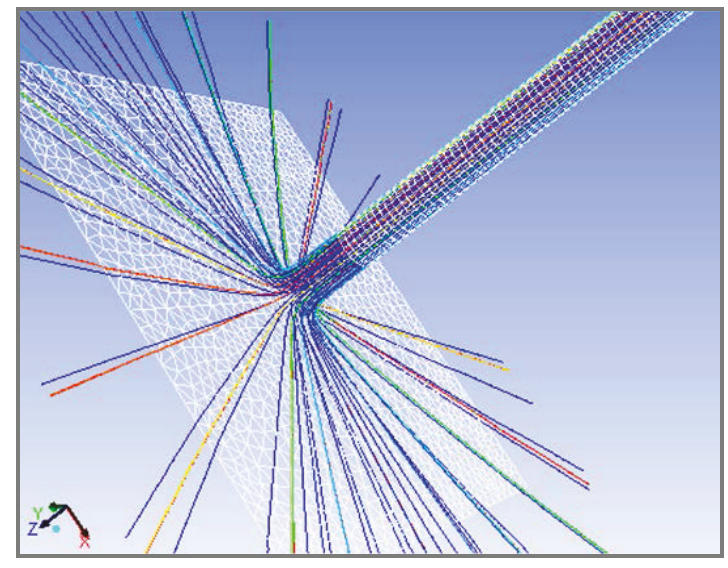

Figure 4: Path lines of air flow impinging on surface

Four combinations of mesh and model were used in the simulation created to evaluate their accuracy against the experimental data set. Two different mesh resolutions were used, both using a wall inflation method normal to the target plate. This method yields high resolution measurements in the y direction - resolution parallel to the plate is not critical for calculating near-wall flow. Freestream velocity Freestream close to the surface is computed by running an initial simulation with a 1st cell height of $1 \mathrm{~mm}$ with the result used to properly calculate the revised required height of the first cell.

The two methods used had a y+ (dimensionless wall distance - equation 6) value of 1 and 15 respectively with calculated values of the 1 st cell height y of $1.0 \mathrm{e}-004 \mathrm{~mm}$ and $1.2 \mathrm{e}-003 \mathrm{~mm}$ respectively (Equation 6.) Cell counts for each method are shown in table 1 - values of $<100,000$ were desired due to constraints on computational resources.

All simulations use the Realizable k- $\varepsilon$ model due to its effectiveness in modelling the spreading of jets [18] 
Table 1: Model matrix with reference codes

\begin{tabular}{clccc}
\hline CODE & Model & Y+ & First Cell Height & Cell Count \\
\hline EWT1 & Enhanced Wall Treatment & 1 & $1.0 \mathrm{e}-004 \mathrm{~m}$ & 71,609 \\
EWT15 & Enhanced Wall Treatment & 15 & $1.2 \mathrm{e}-003 \mathrm{~m}$ & 56,614 \\
STWF & Standard Wall Function & 15 & $1.2 \mathrm{e}-003 \mathrm{~m}$ & 56,614 \\
SCWF & Scalable Wall Function & 1 & $1.0 \mathrm{e}-004 \mathrm{~m}$ & 71,609 \\
ERCO & Results of Cooper et al & $\mathbf{n} / \mathbf{a}$ & $\mathbf{n} / \mathbf{a}$ & $\mathbf{n} / \mathbf{a}$ \\
& (Practical Experiment) & & &
\end{tabular}

\section{THEORY}

Simulating the entire spray painting process as well as its individual components has been completed many times with success in predicting deposition rates [2,5,6,9-11] however improvements are still required by industry to continue to reduce variability. As discussed, this study is concerned with one component of this problem - jet impingement on a surface.

\subsection{JeT IMPINGEMENT \& NeAR WALL FLOW}

This study investigates a single component of the larger air flow and droplet model required to accurately model spray paint deposition. A single jet impinging on a flat surface is a resultant of the high velocity central stream used for atomization in many type of nozzle, however similar effects can be created by other atomizing methodologies. The flow (and deposition) of droplets is highly sensitive to the effects of wall-jet interactions and needs to be accurately modelled. Where the air hits the surface it will create a high velocity (relative to the surrounding air,) flow parallel to the surface which will itself be composed of a number of different layers with differing behaviour. When simulation the region including the jet and surrounding air it is safe to assume a complex turbulent flow field (Reynolds numbers in the order of $10^{4}-10^{5}$ ) however a re-lamination process occurs near the wall (in the boundary region,) as Reynolds numbers drop below the turbulent threshold. The boundary layer $\delta$ is the region of flow near to the wall where the flow velocity u slows from the free stream velocity $U_{\infty}$ to $u=0$ at the wall and exists due to the effects of wall friction and fluid viscosity; the boundary layer is defined as the region where $\mathrm{u}=0$ to $\mathrm{u}=0.99 \mathrm{U} \infty$.

Modelling the region in and around the boundary layer properly creates additional challenges due to the complexity of flow in this region, the layer being further differentiated into three zones of differing behaviour.

Laminar sub layer $\delta_{s}$ : A very thin layer of laminar flow caused by the no-slip condition at the fluid wall interface. The thickness of the laminar sub layer is proportional to the dynamic viscosity of the fluid and inversely proportional to the free stream velocity ( $\&$ turbulence level, as turbulence is also proportional to free stream velocity.) (Equation 1)

$$
\delta_{s} \propto \mu \frac{1}{v_{w}}
$$

Where $\delta_{s}=$ Thickness of laminar sub layer, $\mu=$ Dynamic viscosity, $U_{\infty}=$ Freestream velocity

Buffer Layer/Blending Layer: Where flow can neither be described as fully laminar or fully turbulent.

Fully Turbulent Region: Flow is fully turbulent but velocity is less than $U_{\mathrm{se}}$ 
The relative size of each of the regions is well predicted by 'The law of the Wall' $[12,21]$.

CFD simulation of paint and other particle sprays is normally accomplished using the k- $\varepsilon$ model which has proven accuracy in modelling spreading of sprays and jets, however it relies heavily on an assumption of isotropy in the flow field which does not occur in the laminar sub layer and buffer zone. Accurate modelling of this region (and in effect, accurate spray paint modelling,) depends on being able to adequately compensate for this anisotropy.

\subsection{Wall Functions, Models \& Mesh Design}

Two different methodologies exist for resolving the boundary layer problem - wall functions or near wall modelling (figure 5,6).

i) Wall Functions: These are semi-empirically derived formulae which are used to 'bridge' the viscosity affected region from the wall to the fully turbulent region and do not require mesh resolution of a level smaller than the viscous sub layer.

ii) Near Wall Model Approach: This approach requires generating a mesh with resolution of smaller than the viscous sub layer to allow turbulence calculations to function properly.

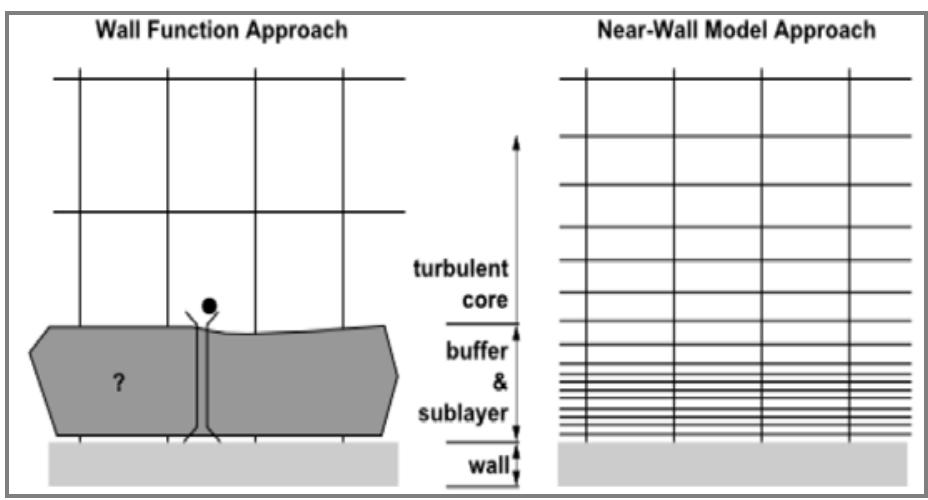

Figure 5: Near wall modelling methodology

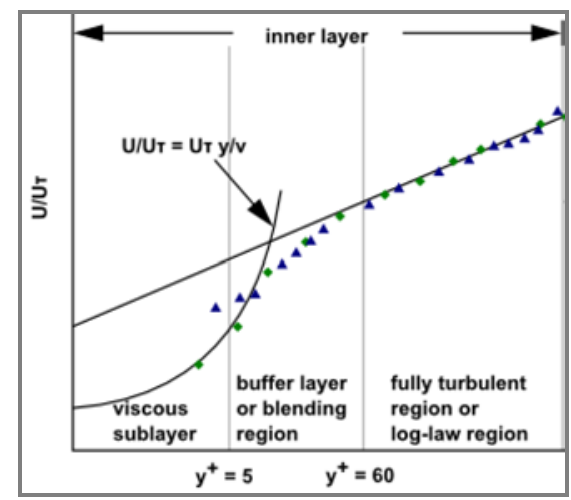

Figure 6: $\mathrm{y}^{+}$values in the boundary layer

Developing a mesh of the required resolution for the method being employed is necessary to produce accurate results. One method normally applied is using the dimensionless value known as $\mathrm{y}^{+}$. As shown in equation 1 , the thickness of the boundary layer is dependent on the freestream flow velocity and the fluid viscosity calculating the boundary layer size as a function of these gives a generalizable dimensionless value. The $y^{+}$ value can be used to describe positions within the boundary layer. Figure 6 shows the relative positions of each of the sub layers within the boundary layer in terms of $y^{+}$. Equations 2-6 from [12] show the calculation of the thickness of the $1^{\text {st }}$ mesh cell using the desired value of $y^{+}$.

$$
\begin{aligned}
& R e=\frac{\rho U_{\text {frastream }} L_{\text {boundary }}}{\mu} \\
& C_{f}=\left[2 \log _{10} R e_{x}-0.65\right]^{-2.3} \quad \text { where } R e_{x}<10^{9} \\
& \tau_{W}=C_{f} \frac{1}{2} \rho U_{\text {freastream }}^{2} \\
& u_{*}=\sqrt{\frac{\tau_{W}}{\rho}} \\
& y=\frac{y^{+} \underline{\mu}}{\rho u_{*}}
\end{aligned}
$$


Where $\mathrm{Re}=$ Reynolds Number,$\rho=$ fluid density, Freestream $=$ Freestream velocity above the boundary layer, $B_{\text {oundary }}=$ Distance downstream from point calculated to start of boundary layer, $\mu=$ dynamic viscosity, $C_{f}=$ skin friction coefficient, $\tau=$ wall shear stress, $u_{*}=$ Friction velocity, $y=$ wall distance, $y^{+}=$Dimensionless wall distance

It is further necessary to ensure that the number of cells covering the boundary layer is in excess of 15 (with 20 being ideal.) The depth of the boundary layer is difficult to estimate properly beforehand as it requires the freestream velocity to be computed first. After initial computation, the centre-line of the boundary layer can be identified as the point where turbulent viscosity reaches its local maxima with the total boundary layer thickness being twice this distance from the wall.

\subsection{VALIDATION OF RESULTS}

Most studies of impinging jet flow relate to cooling and heating applications, however the principles and theory used are the same. As long as results can be generalized in terms of inlet diameter, distance to plate etc. then the results obtained from previous experiments involving such jet impingement can be used to validate CFD data. In this report data collected in previous experiments [3,8], published together as experimental data set [20] is used for experimental validation. The combined data collected from these experiments provides mean velocity, normal stresses and turbulent shear stresses across h/D ratios of 2 and 6 and $r / D$ ratios of 0 to 3 in increments of 0.5 , although this paper is concerned only with comparison of velocity between simulation and experiment.

\section{Results}

The results achieved using the Fluent CFD software are presented here along with those from previous experiment $[17,19]$ for comparison with practically obtained experimental results. Where direct comparison is not possible, comparisons are presented in their generalized form - i.e. distances in terms of ratio to inlet diameter.

Figure 7a shows the velocity profile from directly above the target surface, with the image showing stagnation flow in the central area. Air flow then moves away from the central area, spreading evenly in all directions. Figure $7 \mathrm{~b}$ shows flow parallel to the surface with the magnified image (figure $7 \mathrm{c}$ ) showing the velocity drop to 0 at the wall surface (viscous sub layer.)
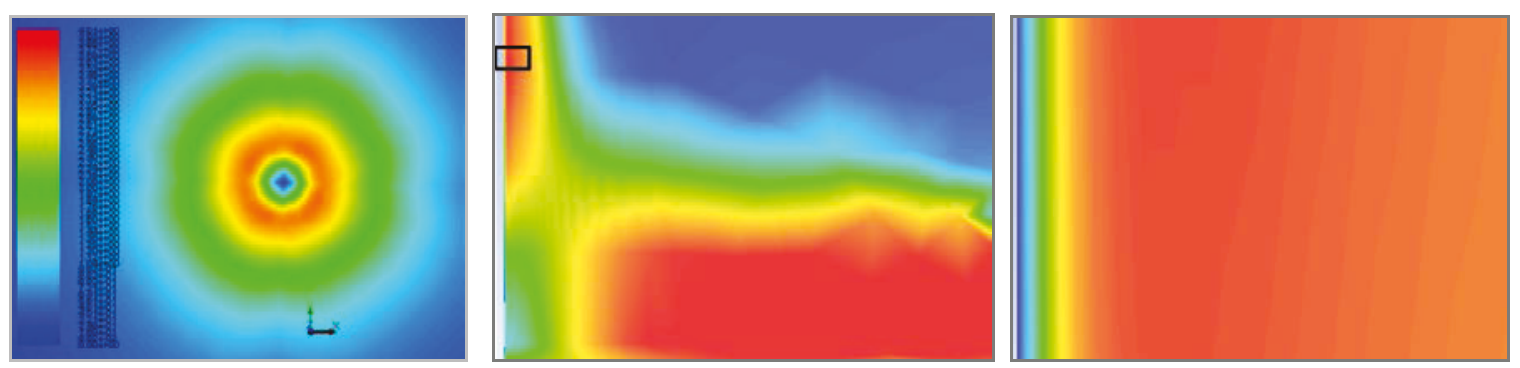

Figure 7a (left): Velocity distribution from above the jet at $1 \mathrm{~mm}$ above the target surface

Figure $7 b$ (centre): Jet impinging on target (plate at left of picture,) showing central jet and freestream wall flow Figure 7c (right): Magnified figure $7 b$ at wall showing viscous sub layer, buffer, log law and freestream regions

The velocity profile from the centre of the jet to $r / D=3$ is shown in figure 8 . Velocity is normalised to $U$ over Maximum freestream velocity in each case. Figure 9 displays velocity at $r / D=1.5$, normalised for freestream velocity and horizontal displacement $r$. Both charts show the four models simulated along with the experimental [20] data (labelled ERCO) for comparison. 


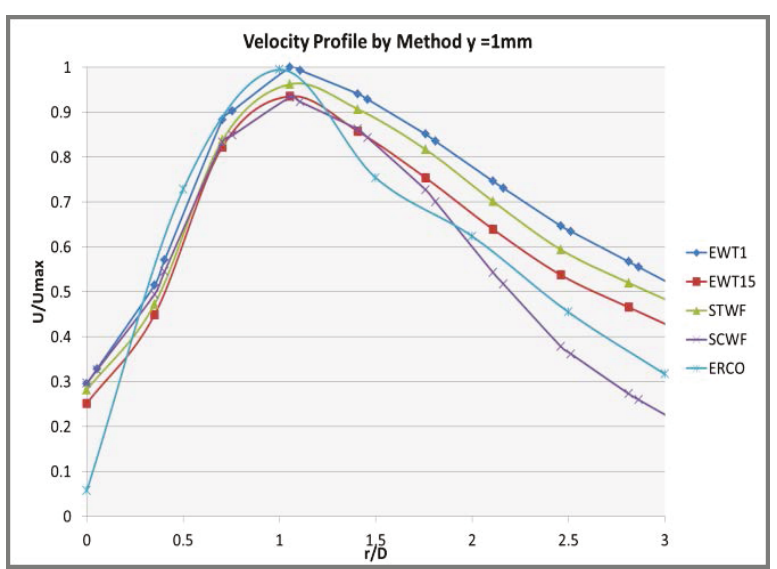

Figure 8: Scaled velocity $U$ at $y=1 \mathrm{~mm}$ for $r / D<=3$.

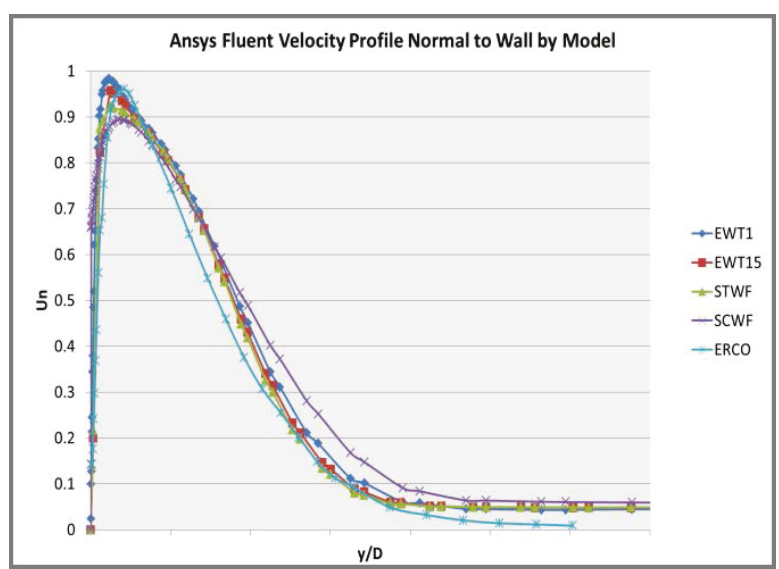

Figure 9: Scaled velocity profile at $r / D=1.5$.

Scaled velocity profiles in figure 10 show velocity as a function of $y / D$ at 5 distances (r/D) from the jet using the dataset obtained from the enhanced wall treatment where $y+=1$. Figure 11 shows the results for the same $r / D$ values at the same locations obtained from the experimental data set [20].
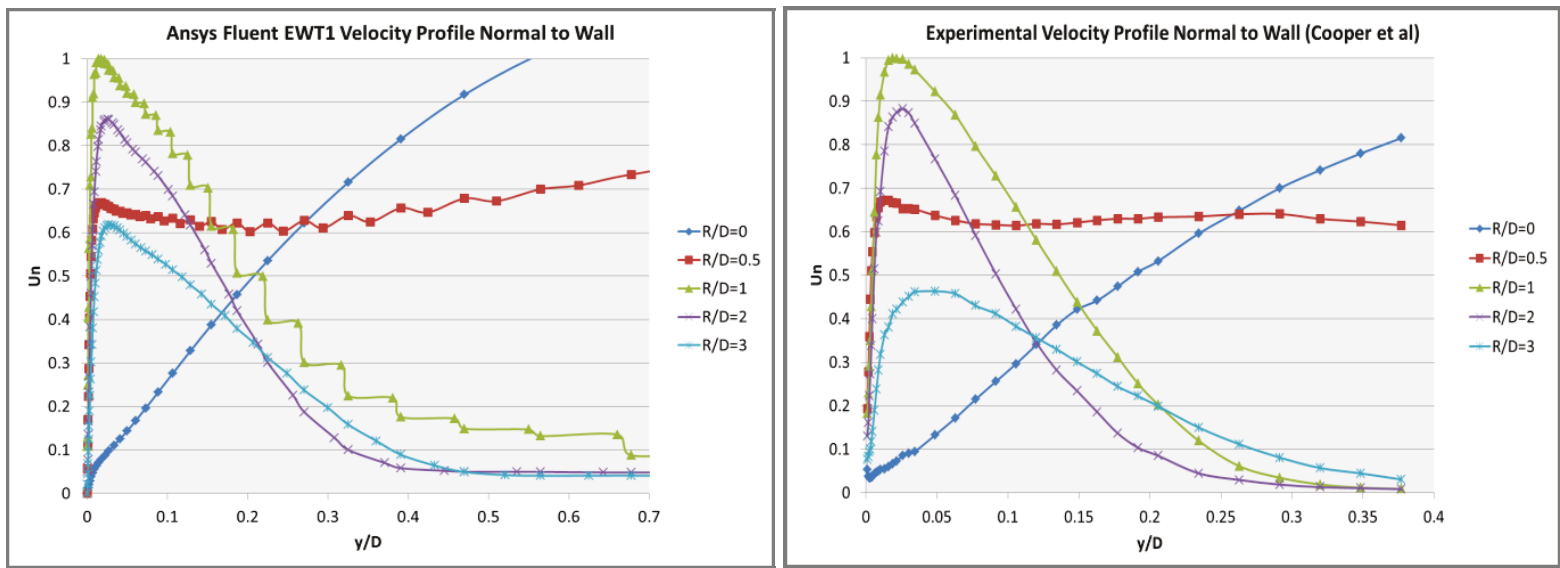

Figure 10: Velocity magnitude from EWT model with $\mathrm{Y}^{+}=1$ Figure 11: Velocity magnitude from ERCOFTAC results [20]

\section{DISCUSSION}

The results detailed in the previous section were obtained with the aim of validating a CFD simulation of a simple impinging jet against existing experimental data while assessing the effectiveness of a number of different mesh designs and models. As discussed, the main interest of the study is with validation of velocity profiles and it is these results that will be discussed here. To better understand the flow pattern around the jet, figures $7 a-7 c$ can be considered. The main phenomena influencing the flow pattern are the stagnation point (figure 7a) where velocity drops to zero and pressure is at its highest (From the Bernoulli equation,) in addition to the wall bounded flow (figure 7b,) and the viscosity affected laminar region shown in figure 7c; proper simulation of the air flow should provide similar results in these regions to that of practical experiment.

Comparing two 3 dimensional data sets numerically is challenging, however reducing the problem to a series of 1 dimensional tests is useful to show commonality or divergence. The velocity measurements in figure 8 are taken at a height of $1 \mathrm{~mm}$ to compare with the experimental results of $[17,19]$.This height is within the boundary layer but outside of the viscous sub layer, meaning the results for each fluent model should not be overly sensitive to the mesh resolution. All models show agreement with the experimental data $[17,19]$ up to 
$r / D=1$ with the results diverging as radial distance from the jet increases. In each case except the scalable wall function, the CFD models produce a higher than experimental scaled velocity at $1 \mathrm{~mm}$ height. Figure 9 shows the velocity profile as $y$ increases (normal to the wall,) at $r / D=1.5$. At this point the wall bounded flow is fully developed, avoiding the effects of the stagnation area and dissipating flow that occurs as $r / D$ increases. Scaled velocities at this point show strong agreement with experimental data with only the scalable model overestimating velocities as distance away from the wall increase. When examining the full data set, the closest results to experimental values were provided by the enhanced wall treatment with mesh resolving the viscous sub layer (EWT1) matching with ANSYS recommendations.

Figure 10 and 11 show the comparisons between the EWT1 simulation results and the experimental data. The curves plotted again represent velocity at a scaled distance from the wall surface with each curve showing the behaviour at a different scaled radial distance from the jet. Scaled results again show excellent agreement in and around the boundary layer $(y / D=0.05)$ with the boundary layer estimated at $\sim 4 \mathrm{~mm}$ using the turbulent kinetic intensity maximum. As $y / D$ increases, the CFD model predicts higher velocities than recorded [20]; however as this study is primarily interested in the flow in the boundary layer(where velocity is significantly higher than the surrounding air,) the results are a good predictor of wall bounded flow in this region.

\section{CONCLUSIONS}

In each case, the Fluent models used provided excellent simulation of the behaviour of the air flow in and around the boundary region. While the coarser mesh models use wall functions to estimate the viscous and buffer layer flows and consequently produced much lower resolution data in the wall region, their results compared to the near wall model approach were excellent. Scaled results show strong agreement between the experimental results and the CFD data with the general flow behaviour being described accurately - best shown in the results of the enhanced wall treatment at $y+=1$ (figure 10 \& 11). Further refinements in the models used are required to better resolve the air flow as radial distance from the centre increases. Further experiment will be based on the enhanced wall treatment and will focus on recreating the existing experimental data discussed here and then further proving generality across a range of inlet diameters. This work will then be used in the continued development of a new paint deposition model.

\section{ACKNOWLEDGMENTS}

The authors would like to thank MTE Ltd and Dr Keith Bell for their invaluable and continued support during this project.

\section{REFERENCES}

[1] Domnick, J., Scheibe, A. \& Ye, Q., n.d. The Electrostatic Spray Painting Process with High-Speed Rotary Bell Atomizers: Influences of Operating Conditions and Target Geometries, Stuttgart: Fraunhofer-Institute for Manufacturing Engineering \& Automation.

[2] Lin, J., Qian, L., Hongbin, X. \& Chan, T. L., 2009. Effects of operating conditions on droplet deposition onto surface of atomization impinging spray. Surface \& Coatings Technology, Volume 203, pp. 1733-1740.

[3] Yao, S., Zhang, J. \& Fang, T., 2010. Effect of Viscosities on the Instability of Sprays from a Swirl Atomizer. Cincinatti, S.n.

[4]: Sterling, A. M. \& Sleicher, C. A., 1975. The Instability of Capiliary Jets. Journal of Fluid Mechanics, 68(3), pp. 477-495.

[5] Prabhakaran, V. S., Sivakumar, D. \& Tharakan, T. J., 2009. Effect of temperature on atomization in gas centered coaxial injection systems, Bombay: Indian Institute of Technology.

[6] Fernando, R. H., Xing, L. L. \& Glass, J. E., 2000. Rheology parameters controlling spray atomization and roll misting behaviour of waterborne coatings. Process in Organic Coatings, Volume 40, pp. 35-38.

[7] Godfrey, J. C. \& Edwards, M. F., 1990. A Power-Law Model of Thixotropy. s.l., Elsevier/Springer. 
[8] Ye, Q., Shen, B., Tiedje, O. \& Domnick, J., 2011. Investigations of spray painting processes using an airless spray gun. s.I., ILASS Europe.

[9] Viti, V. \& Kulkarni, J., 2008. CFD Analysis of the Electrostatic Spray Painting Process. Orlando, ILASS Americas.

[10] Ejim, C. E., Rahman, M. A., Amirfazli, A. \& Fleck, B. A., 2010. Effects of liquid viscosity and surface tension on atomization in two-phase, gas/liquid fluid coker nozzles. Fuel, Volume 89, pp. 1872-1882.

[11] Blum, S., Henkel, N., Lehnhauser, T. \& Neubauer, H.-J., 2009. Optimization of process parameters for automotive paint application. Weimar, Dynardo.

[12] Yu, S. \& CAO, L., 2011. Modeling and Prediction of Paint Film Deposition Rate for Robotic Spray Painting. Beijing, IEEE.

[13] Qiaoyan, Y. \& Gui, X., 2006. Numerical simulation of turbulent boundary for stagnation-flow in spray-painting processes. Gottingen, Conference on Boundary and Interior Layers.

[14] T.S O'Donovan and D.B Murray, "Jet impingement heat transfer - Part 1 Mean and root-mean-square heat transfer and velocity distributions", International Journal of Heat and Mass Transfer, issue 50, pp3291-3301, 2007

[15] M.Behnia, S.Parneix and P.Durbin, "Simulation of jet impingement heat transfer with the $k-\varepsilon-v^{2}$ model", Center for Turbulence Research, Annual research Briefs 1996

[16] Y Yamane, Y Ichikawa, M Yamamoto and S Honami, "Effect of Injection Parameters on Jet Array Impingement Heat Transfer", Interantional Journal of Gas Turbine, Propulsion and Power Systems, Volume 4, Issue 1, 2012

[17] J W Baughn and S Shimizu, "Heat Transfer Measurements From a Surface With Uniform Heat Flux and an Impinging Jet", J Heat Transfer, vol.111, issue 4 pp1096-1098, 1989.

[18] ANSYS. (n.d.). Fluent Theory Guide.

[19] D. Cooper, D.C. Jackson, B.E. Launder, G.X. Liao, Impinging Jet Studies for Turbulence Model Assessment, Part I: Flowfield Experiments, Int. J. Heat Mass transfer, Vol. 36, issue 10, pp 2675-2684, 1993.

[20] European Research Community on Flow, ERCOFTAC Database. Case 25 Cooper et al Open dataset http://cfd.mace.manchester.ac.uk/cgi-

bin/cfddb/prpage.cgi?25\&EXP\&\&database/cases/case25\&cas25_head.htm/\&cas25_desc.htm/\&cas25_meth.htm/\&ca s25_data.htm/\&cas25_refs.htm/\&cas25_rsol.htm/\&1\&1\&1\&1\&1\&unknown

[21] Schlichting, H., \& Gersten, K.. Boundary-Layer Theory. New York: McGraw Hill. (2000) 